\title{
STUDY OF VARIATIONS IN MUSCULAR BRANCHES OF RADIAL NERVE IN AXILLA AND POSTERIOR COMPARTMENT OF ARM
}

\author{
Raghavendra D R ${ }^{* 1}$, Nirmala $D^{2}$, Maveshettar G F ${ }^{3}$. \\ ${ }^{{ }_{1}}$ Assistant Professor, Department of Anatomy, Kamineni Institute of Medical Sciences, Narketpally, \\ Telangana, India. \\ 2,3. Professor, Department of Anatomy, J J M Medical College, Davangere, Karnataka. India.
}

\section{ABSTRACT}

Background: Radial nerve originates from posterior cord of brachial plexus at axilla. It supplies extensor muscles of upper limb.

Objectives: To know the variations in muscular branches of radial nerve in axilla and posterior compartment of arm.

Methods: Dissection was done on 44 upper limbs from embalmed cadavers and 6 upper limbs from embalmed dead fetuses in the Department of Anatomy, J J M Medical College, Davangere. Dissection of Radial nerve and its branches in the axilla and posterior compartment of the arm was carried out according to Cunningham's manual of practical anatomy.

Results: The site of origin of nerve to long head of tricep (N-LHT) was axilla in 48 specimens (96\%) and lower triangular space( LTS) in 2 specimens (4\%). The site of origin of nerve to lateral head of tricep( N-LTHT) was radial groove(RG) in 49 specimens (98\%) and lower triangular space(LTS) in 1 specimens (2\%). The site of origin of nerve to medial head of tricep -ulnar collateral nerve(UCN) was axilla in 38 specimens (76\%) and lower triangular space(LTS) in 12 specimens (24\%). The site of origin of nerve to medial head of tricep-nerve to anconeus(NA) was radial groove(RG) in 49 specimens (98\%) and lower triangular space(LTS) in 1 specimens (2\%). The additional branches to long head of tricep(LHT) was found in 20 specimens (40\%). The additional branches to lateral head of tricep( LTHT) was found in 10 specimens (20\%). The additional branches to medial head of tricep(MHT) was found in 7 specimens (14\%).

Interpretation and Conclusion: The present study is important for Surgeons, Orthopedicians, and Neurophysicians as it provides the knowledge of variations in muscular branches of radial nerve in axilla and posterior compartment of arm to prevent possible complications.

KEY WORDS: Radial Nerve, Triceps, Radial Groove. Wrist Drop.

Address for Correspondence: Dr Raghavendra D R, Assistant Professor, Department of Anatomy, Kamineni Institute of Medical Sciences, Narketpally, 508254, Telangana. India.

E Mail: dr.raghavendra18@gmail.com

Access this Article online

Quick Response code

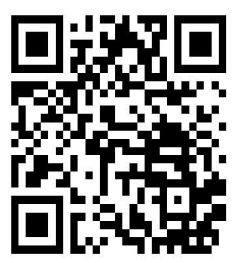

DOI: $10.16965 /$ ijar.2018.445

Journal Information

\section{International Journal of Anatomy and Research}

ICV for 2016 ISSN (E) 2321-4287 | ISSN (P) 2321-8967

90.30 https://www.ijmhr.org/ijar.htm

DOI-Prefix: https://dx.doi.org/10.16965/ijar

$( c c ) \longdiv { B Y - M B - S A }$

\section{Article Information}

Received: 10 Dec 2018

Peer Review: 11 Dec 2018

Revised: None
Accepted: 20 Jan 2019

Published (O): 05 Feb 2019

Published (P): 05 Feb 2019

\section{INTRODUCTION}

Nerve fibres transmit messages (nerve impulses) either from the central nervous system to the various structures of the body or from these structures to the central nervous system. Motor nerve fibres pass to the muscles to make them contract. Radial nerve is the continuation of the posterior cord of the brachial 
plexus in the axilla. Here it gives off the nerve to the long head of triceps and then passes in to the arm posterior to the brachial artery. Almost immediately it gives off a branch to the medial head of triceps (which accompanies the ulnar nerve into the posterior compartment), then passes inferolaterally into the groove for the radial nerve on the posterior surface of the humerus winding spirally round the posterior surface of the humerus with the profunda brachii artery, in contact with the periosteum. In the groove, the nerve gives off branches to the lateral head and a long slender branch which descends through the medial head of triceps to the muscle anconeus distal to the lateral epicondyle. The nerve then pierces the lateral intermuscular septum and descends in the anterior compartment between brachialis (medially) and brachioradialis and extensor carpi radialis longus (laterally). It divides in to superficial and deep branches[1].

Muscular branches of radial nerve in axilla and posterior compartment of arm: Muscular branches in the axilla and posterior compartment of arm supply triceps and anconeus in medial and posterior groups. Nerve to long head of triceps and Nerve to medial head of triceps given in axilla. Medial muscular branches arise from the radial nerve on the medial side of the arm. They supply the medial and long heads of triceps.With the profunda brachii artery radial nerve inclines dorsally, passing through the triangular space. Here it supplies the long head of triceps. The branch to the medial head is a long, slender filament which, lying close to the ulnar nerve as far as the distal third of the arm, is often termed the 'ulnar collateral nerve'[2].

Nerve to lateral head of triceps and Nerve to medial head of triceps and anconeus are given in radial groove. Radial nerve spirals obliquely across the back of the humerus, lying posterior to the uppermost fibres of the medial head of triceps which separate the nerve from the bone in the first part of the spiral groove. Here it gives off a muscular branch to the lateral head of triceps and a branch which passes through the medial head of triceps to anconeus. A large posterior muscular branch arises from the nerve as it lies in the humeral groove. It divides to supply the medial and lateral heads of triceps and anconeus, that for the latter being a long nerve which descends in the medial head of triceps and partially supplies it; it is accompanied by the middle collateral branch of the profunda brachii artery and passes behind the elbow joint to end in anconeus[2].

The radial nerve is also known as the "great extensor nerve" due to its innervation of the triceps, wrist and finger extensors[3].The radial nerve can be damaged in the axilla and in the spiral groove[4].The radial nerve, which supplies all of the extensor muscles of the upper limb, passes diagonally around the posterior surface of the middle of the humerus in the radial groove. The most common site of radial nerve injury is in the radial groove[5].The radial nerve is at risk during the posterior plating of the humerus[6]. Identification of radial nerve is necessary during the posterior approach to the humerus in an effort to maintain its integrity and to lessen the possibility of neurologic compromise[7].

Hence the study of the radial nerve is undertaken, the results of this study may be useful in various surgical procedures in axilla and posterior compartment of the arm and evaluating the radial nerve injuries and their management for Surgeons, Orthopedicians, and Neurophysicians.

\section{MATERIALS AND METHODS}

The specimens for this study were obtained from embalmed cadavers and embalmed dead fetuses from the Department of Anatomy, J.J.M. Medical College, Davangere. 50 upper limbs were procured from the Department of Anatomy. Among them 6 specimens were belong to embalmed dead fetuses. Study conducted over a period of two years. Dissection of Radial nerve and its branches in the axilla and posterior compartment of the arm was carried out according to Cunningham's manual of practical anatomy

The upper limb in each supine cadaver was abducted and laterally rotated. Skin was incised from manubrium sterni to both xiphoid process of sternum as well as acromion process of scapula. Further, the skin was incised from xiphoid process extending upwards and laterally, along the floor of axilla, to the middle of the arm. Incision was further extended from middle of the arm up to apex of cubital fossa. 
The skin and superficial fascia were reflected from the deep fascia by blunt dissection. The deep fascia was then incised to expose muscles. The pectoralis major and minor muscles were reflected laterally to expose axilla. The axilla was dissected by removing loose connective tissue and fat. The axillary fascia was then incised and lymph nodes were removed to expose the cords of brachial plexus. The coracobrachialis and short head of biceps brachii muscle were then exposed. Axillary artery and vein and the large nerves surrounding them were exposed. Radial nerve identified behind the axillary artery. The long head of triceps brachii was exposed. Muscular branches of radial nerve in axilla were identified and cleaned. Any additional muscular branches, if present were noted.

The cadaver was then pronated to expose the posterior aspect of the arm. The skin and superficial fascia were reflected from deep fascia by blunt dissection. The deep fascia over triceps brachii was incised. Long and lateral heads of the triceps were identified and cleared. Lower triangular space identified and cleared. Radial nerve identified in axilla and traced through lower triangular space as far as triceps. Lateral head of the triceps muscle divided and reflected to expose the radial nerve and profunda brachii artery in the groove for the radial nerve on the back of the humerus. Medial head of triceps identified inferior to the groove. Muscular branches of radial nerve were identified and cleaned. Any additional muscular branches, if present were noted.

Site of origin of muscular branches and presence of additional branches were noted. Photograph of each specimen was taken after dissection with digital camera.

\section{RESULTS}

The site of origin of nerve to long head of tricep ( N-LHT) was axilla in 48 specimens(96\%) and lower triangular space( LTS) in 2 specimens (4\%). The additional branches to long head of tricep( LHT) was found in 20 specimens(40\%). The origin of additional branches to long head of tricep(LHT) was from nerve to long head of tricep( $\mathrm{N}$-LHT) in 16 specimens (80\%), and from radial nerve( $R N)$ in 4 specimens(20\%). The site of origin of additional branches to long head of
tricep(LHT) was axilla in 12 specimens(57.1\%) and lower triangular space(LTS) in 9 specimens (42.9\%).

The site of origin of nerve to lateral head of tricep(N-LTHT) was radial groove(RG) in 49 specimens(98\%) and lower triangular space( LTS) in 1 specimen (2\%). The additional branches to lateral head of tricep( LTHT) was found in 10 specimens(20\%). The origin of additional branches to lateral head of tricep( LTHT) was from radial nerve( $R N)$ in 6 specimens (60\%), from nerve to lateral head of tricep (N-LTHT) in 2 specimens (20\%) and from lower lateral cutaneous nerve of arm (LLCNA) in 2 specimens (20\%). The site of origin of additional branches to lateral head of tricep( LTHT) was radial groove (RG) in 10 specimens (100\%).

The site of origin of nerve to medial head of tricep-ulnar collateral nerve( UCN) was axilla in 38 specimens (76\%) and lower triangular space( LTS) in 12 specimens (24\%).The site of origin of nerve to medial head of tricep-nerve to anconeus ( NA) was radial groove(RG) in 49 specimens (98\%) and lower triangular space( LTS) in 1 specimen (2\%). The additional branches to medial head of tricep( MHT) was found in 7 specimens (14\%). The origin of additional branches to medial head of tricep( $\mathrm{MHT}$ ) was from radial nerve(RN) in 7 specimens (100\%), The site of origin of additional branches to medial head of tricep( $\mathrm{MHT}$ ) was radial groove(RG) in 7 specimens (100\%).

Fig. 1: Radial nerve branches in axilla and lower triangular space(LTS).

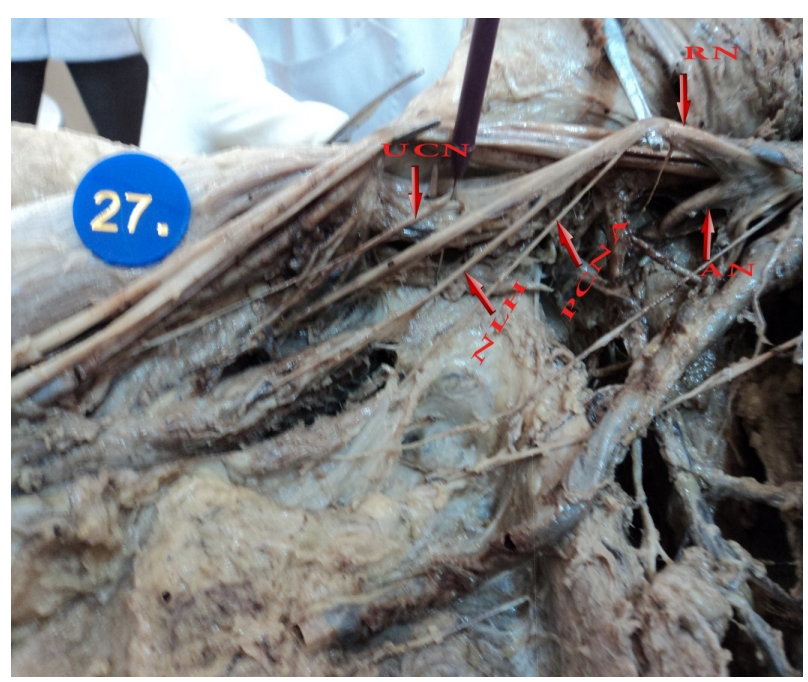

Ulnar collateral nerve(UCN) taking origin at LTS.( RN:Radial nerve, AN: Axillary nerve, PCNA:Posterior cutaneous nerve of arm, NLH:Nerve to long head of tricep) 
Fig. 2: Radial nerve branches in axilla and lower triangular space(LTS).

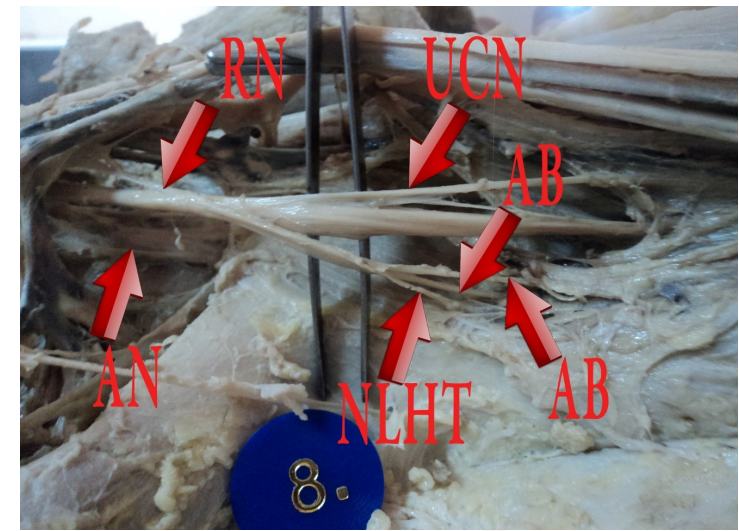

Additional branches $(A B)$ to long head of tricep from NLHT.( RN:Radial nerve, AN: Axillary nerve, NLHT:Nerve to long head of tricep, UCN: Ulnar collateral nerve)

Fig. 3: Radial nerve branches in Posterior compartment of arm.

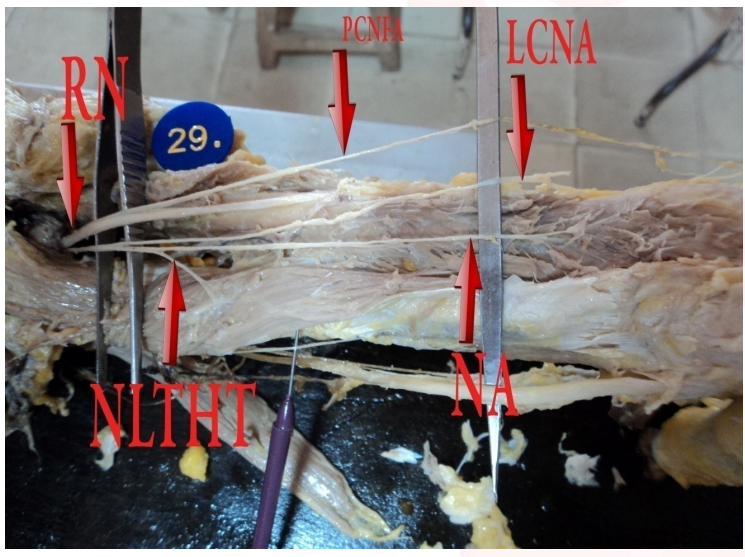

(RN:Radial nerve, NLTHT:Nerve to lateral head of tricep, NA: Nerve to medial head of tricep \& anconeus,LCNA: Lower lateral cutaneous nerve of arm,PCFNA:Posterior cutaneous nerve of forearm)

Fig. 4: Radial nerve branches in Posterior compartment of arm.

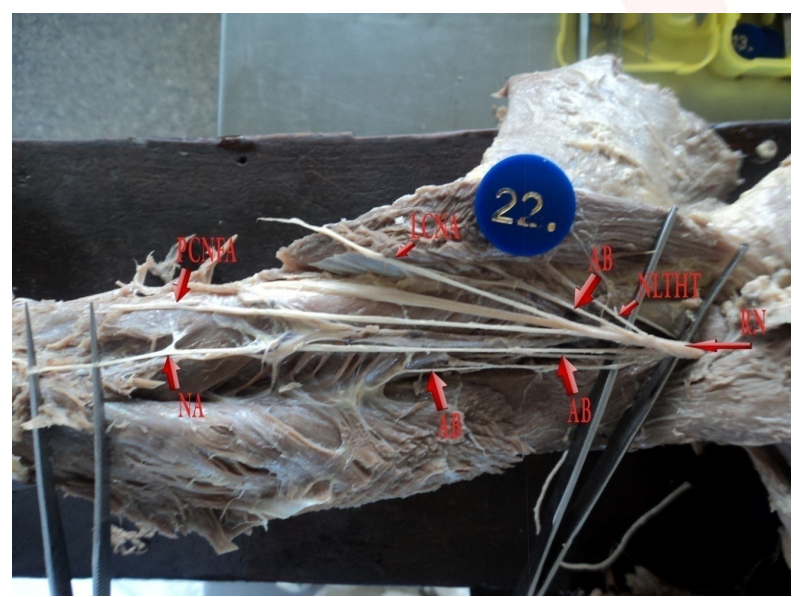

Additional branches $(A B)$ to medial head of tricep from Radial nerve(RN) (NLTHT:Nerve to lateral head of tricep, NA: Nerve to medial head of tricep \& anconeus,LCNA: Lower lateral cutaneous nerve of arm,PCFNA:Posterior cutaneous nerve of forearm)
Table 1: Site of origin of radial nerve branches and additional muscular branches.

\begin{tabular}{|c|c|c|c|c|}
\hline \multirow{3}{*}{ Nerve } & \multirow{2}{*}{\multicolumn{3}{|c|}{$\begin{array}{c}\text { Site of origin of radial nerve } \\
\text { branches } \\
\begin{array}{c}\text { Number of specimen with } \\
\text { percentage }\end{array} \\
\end{array}$}} & \multirow{2}{*}{$\begin{array}{c}\text { Additional muscular } \\
\text { branches }\end{array}$} \\
\hline & & & & \\
\hline & Axilla & LTS & RG & \\
\hline NLHT & 48 (96\%) & $2(4 \%)$ & & $20 \quad(40 \%)$ \\
\hline NLTHT & & 1 (2\%) & $49 \quad(98 \%)$ & $10 \quad(20 \%)$ \\
\hline NMHT & & & & $7 \quad(14 \%)$ \\
\hline UCN & 38 (76\%) & $12(24 \%)$ & & \\
\hline NA & & $1(2 \%)$ & 49 (98\%) & \\
\hline
\end{tabular}

(NLHT-nerve to long head of tricep, NLTHT- nerve to lateral head of tricep, NMHT-nerve to medial head of tricep, UCN-ulnar collateral nerve,NA-nerve to anconeus,LTS-lower triangular space,RG-radial groove)

\section{DISCUSSION}

In the present study, out of 50 specimens, The site of origin of nerve to long head of tricep ( $\mathrm{N}$-LHT) was axilla in 48 specimens(96\%) and lower triangular space(LTS) in 2 specimens (4\%). Stanescu recorded in his study of 33 cadaveric dissections performed to identify radial nerve branching patterns to the triceps brachii, innervation of the long head of the triceps originated in the axilla in $88 \%$ and the brachioaxillary angle in $12 \%$ [8]. The findings of present study is comparable with Stanescu.

The site of origin of nerve to lateral head of tricep( N-LTHT) was radial groove (RG) in 49 specimens (98\%) and lower triangular space(LTS) in 1 specimens (2\%). Stanescu reported, the lateral head was innervated by branches arising in the spiral groove in $70 \%$, the brachioaxillary angle in $24 \%$, which is higher than the present study and the axilla in 6\%[8]. In the present study N-LTHT did not arise in axilla in any of the specimens.

The site of origin of nerve to medial head of triceps-ulnar collateral nerve(UCN) was axilla in 38 specimens(76\%) and lower triangular space(LTS) in 12 specimens (24\%).The site of origin of nerve to medial head of triceps-nerve to anconeus( NA) was radial groove( RG) in 49 specimens (98\%) and lower triangular space(LTS) in 1 specimens (2\%). Johnson mentions that, The branch to the medial head is a long, slender filament which, lying close to the ulnar nerve as far as the distal third of the arm, is often termed the 'ulnar collateral nerve'[2]. 
Present study confirms the course of UCN. Stanescu mentions, Innervation of the medial head originated in the spiral groove in $52 \%$, the brachioaxillary angle in $39 \%$, and the axilla in $9 \%[8]$. The present study confirms medial head of triceps is supplied by the branches arising in axilla, lower triangular space and radial groove. The incidence of branches to medial head of tricep( MHT) arising in the radial groove in the present study is higher than the findings of Stanescu.

Sunderland mentions, all the branches to triceps brachii were distributed through axilla and radial groove, in all the specimens. Anconeus was supplied by the branch to medial head of triceps brachii which descended in the substance of that muscle. In half of the specimens, the lateral head of triceps brachii was innervated before its medial head regardless of the order of branching. In half of the specimens, the lateral head of triceps brachii were supplied by branches leaving the nerve in radial groove. In one third of the specimens, the medial head of triceps brachii were supplied by branches leaving the nerve in radial groove. Majority of them were in contact with the bone[9]. Present study has similar findings. de Seze et al., mentions in their study, branch supplying the long head of triceps brachii arose from the axillary nerve but not from the radial nerve[10]. Present study has no such findings.

The present study also helps in various surgical approaches of open reduction of humerus which may even involve anterior transposition of radial nerve.

\section{CONCLUSION}

The site of origin of nerve to long head of tricep (N-LHT) was axilla in 48 specimens (96\%) and lower triangular space( LTS) in 2 specimens (4\%). The site of origin of nerve to lateral head of tricep( N-LTHT) was radial groove(RG) in 49specimens (98\%) and lower triangular space(LTS) in 1 specimens (2\%). The site of origin of nerve to medial head of tricep -ulnar collateral nerve(UCN) was axilla in 38 specimens (76\%) and lower triangular space(LTS) in 12 specimens (24\%). The site of origin of nerve to medial head of tricep-nerve to anconeus(NA) was radial groove(RG) in 49 specimens (98\%) and lower triangular space (LTS) in 1 specimens (2\%). The additional branches to long head of tricep(LHT) was found in 20 specimens (40\%). The additional branches to lateral head of tricep( LTHT) was found in 10 specimens (20\%). The additional branches to medial head of tricep(MHT) was found in 7 specimens (14\%). The present study found additional muscular branches either from nerve to the muscle itself before entering the muscle or directly from radial nerve and its other branches. The presence of additional muscular branches plays important role in preserving muscular activity during nerve injury.

\section{Conflicts of Interests: None}

\section{REFERENCES}

[1]. Romanes GJ. Cunningham's Manual of Practical Anatomy, Volume 1, upper and lower limbs. $15^{\text {th }}$ ed. New York: Oxford medical pubs.2010.p.4-73.

[2]. Standring S. Gray's Anatomy. The Anatomical Basis of Clinical Practice. In: Johnson D, editor. Pectoral girdle and upper limb. $40^{\text {th }}$ ed. Edinburg : Churchill Livingstone; 2008.p.781-829.

[3]. Chang IT, Hohler AD. Bilateral radial nerve compression(crutch palsy): A case report.J Neurol Neurophysiol 2012; 3:3.

[4]. Snell RS. Clinical Anatomy by Regions. $9^{\text {th }}$ ed. Philadelphia: Wolters Kluwer/Lippincott Williams and Wilkins; 2012.430-431.

[5]. Drake RL, VogI AW, Mitchel AWM. Anatomy for Students. $2^{\text {nd }}$ ed. Edinburg: Churchill Livingstone; 2010.663-774.

[6]. Yakkanti MR, Roberts CS, Murphy JBS, Acland RD. Anterior transposition of the radial nerve-A cadeveric study. Journal of Orthopedic Trauma 2008; 22(10):705-708.

[7]. Seigerman DA, Choung EW, Yoon, RS, Lu M, Frank MA, Gaines LCDRRJ, et al. Identification of the Radial Nerve During the Posterior Approach to the Humerus: A Cadaveric Study. J Orthop Trauma 2012; 26: 226-228.

[8]. Stanescu S, Post I, Ebraheim NA, Bailey AS, Yeasting R. Surgical anatomy of the radial nerve in the arm: Practical considerations of the branching patterns to the triceps brachii. Orthopedics 1996; 19: 311315.

[9]. Sunderland S. The Radial Nerve- Anatomical and Physiological Features. In: Sunderland S, editor. Nerve and Nerve Injuries. $2^{\text {nd }}$ ed. Edinburgh: Churchill Livingstone; 1978; 802-819.

[10]. de Seze MP, Rezzouk J, de Seze M, Uzel M, Lavignolle $B, M i d y D$, et al. Does the motor branch of the long head of triceps brachii arise from the radial nerve? An anatomic and electromyographic study. Surg Radiol Anat 2004; 26: 459-469. 